\title{
Characterization of Uncertainties When Measuring Metal Cutting Temperatures Using Infrared Radiation Thermography
}

\author{
Eric P. Whitenton \\ National Institute of Standards and Technology, Gaithersburg, MD USA 20899-8223
}

\begin{abstract}
There are many error sources when using infrared radiation thermography to measure the temperature distribution of the tool, workpiece, and chip during metal cutting. It is important to understand how these error sources affect the measurement uncertainty. Some are familiar to anyone performing thermography measurements, such as uncertainties in the basic camera calibration. However, metal cutting presents unique measurement challenges due to factors such as the high magnification required, high surface speeds, polarization effects, micro-blackbody effects, and changing emissivity as chips form. This paper presents highlights of the current state of efforts at NIST to catalog and characterize error sources and the resulting uncertainties.
\end{abstract}

Keywords: uncertainty, metal cutting, dual spectrum, high-speed imaging, infrared thermography

\section{INTRODUCTION}

Finite element modeling simulations are an important tool for optimizing the metal cutting process, allowing industry to make parts faster, better, and at less cost. Measurements of the process using thermal ${ }^{[1,2]}$ and visible ${ }^{[3]}$ imaging can be used to improve and verify the accuracy of these simulations. An example of a comparison is shown in Figure 1. The material removed from the workpiece is referred to as a chip. Two of the many types of chips are continuous and segmented chips. A continuous chip is a long ribbon of relatively uniformly deformed material. This contrasts to a segmented chip, which has alternating zones of low and high shear strain. A zone of low strain is called a segment. The zones of high strain between the segments are mechanically weak, so the long ribbons of material tend to break up into short, more manageable ones. Even where the chips do not break completely, there is generally a partial gap between segments.

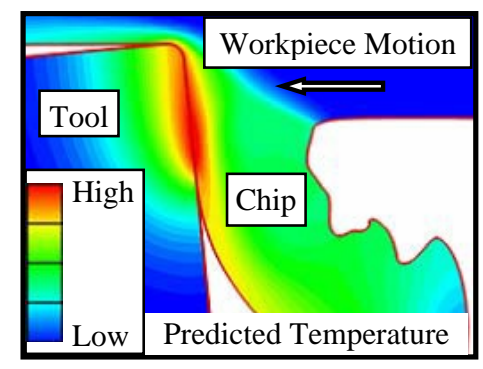

a
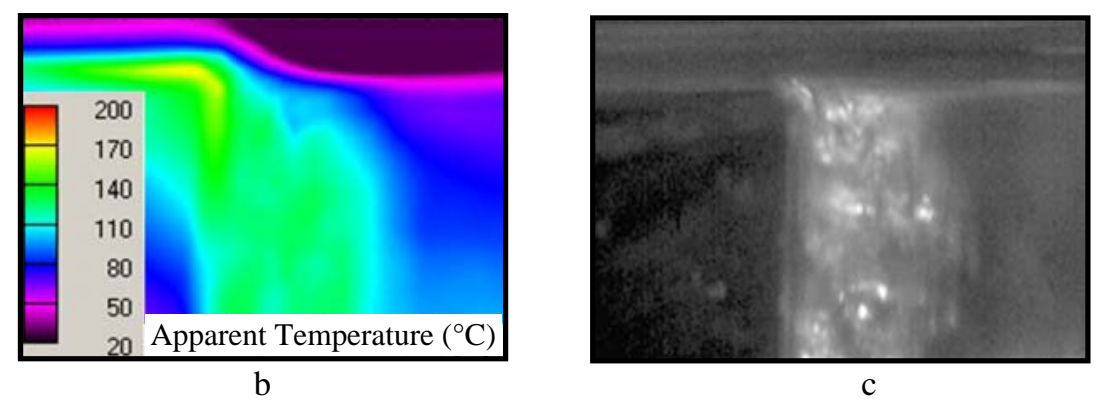

Fig. 1. Finite element models of machining (a) may be compared to high-speed thermal (b) and visible (c) spectrum images of machining to verify the models. The continuous chip is approximately $0.6 \mathrm{~mm}$ thick.

This paper is an official contribution of the National Institute of Standards and Technology and is not subject to copyright in the United States. The National Institute of Standards and Technology does not recommend or endorse commercial equipment or materials, nor does it imply that the equipment or materials shown are necessarily the best available for the purpose. 


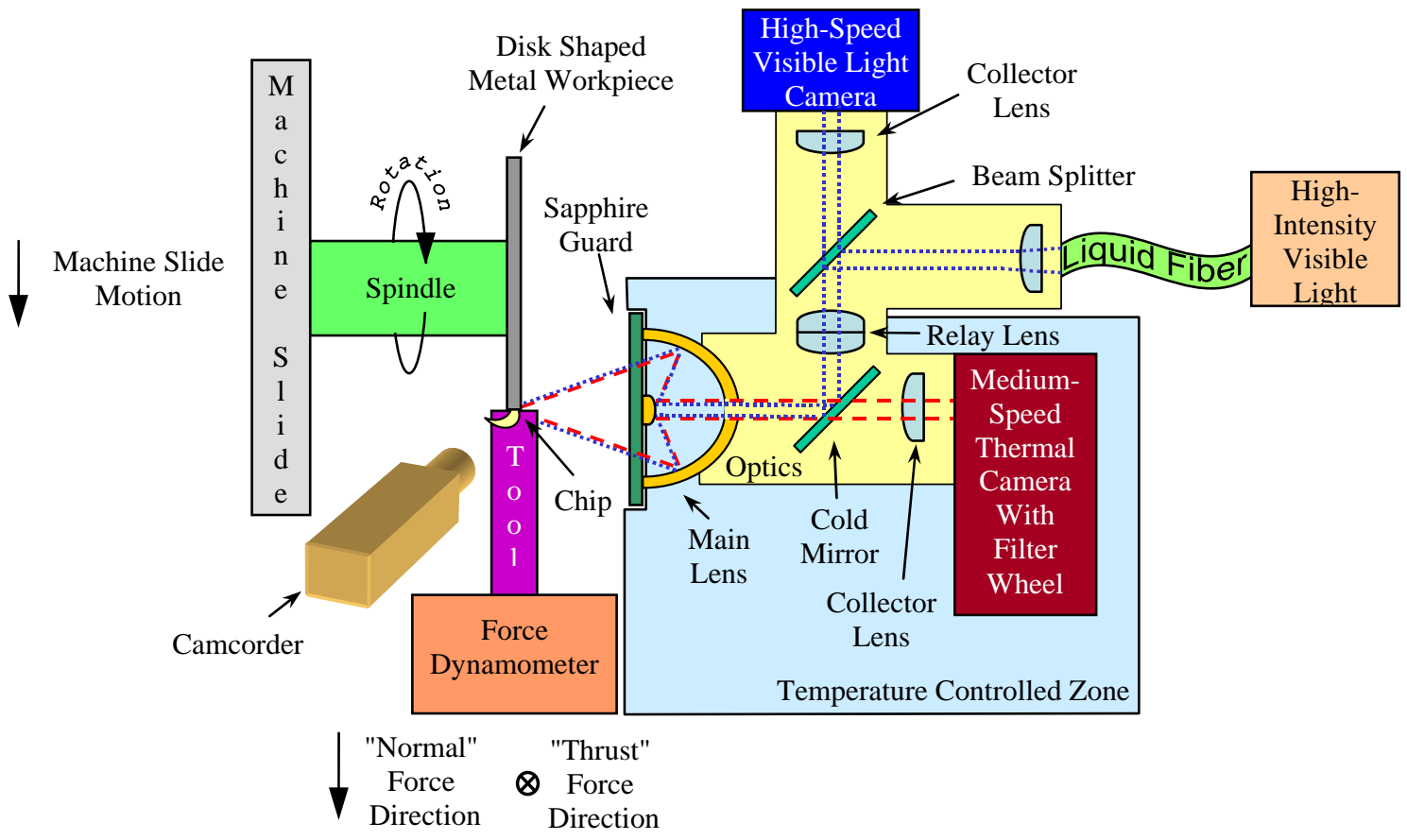

Fig. 2. High-speed, thermal and visible radiation (dual-spectrum) camera system used to measure machining processes.

Figure 2 shows a unique high-speed, thermal and visible radiation (dual-spectrum) camera system developed by the National Institute of Standards and Technology ${ }^{[1,2]}$. It images the side of the chip as the chip is being formed, generally with about a $1 \mathrm{~mm}$ wide field of view. The visible spectrum images are typically acquired at 30000 frames per second, yielding 256 pixel by 128 pixel images. The workpiece and chip are typically traveling between $0.5 \mathrm{~m} / \mathrm{s}$ and $5 \mathrm{~m} / \mathrm{s}$. To minimize motion blur, integration time is adjusted to the shortest possible. The thermal spectrum images are typically acquired at 300 frames per second, yielding 160 pixel by 120 pixel images. Integration time is adjusted to minimize motion blur while maintaining enough sensitivity to image temperatures of interest. At an angle to the dual-spectrum system is a conventional camcorder which images the scene at a low magnification. An example of the camcorder images is shown in Video 1. A data acquisition system records the timing of the three image streams so they may be played back in a synchronized manner.

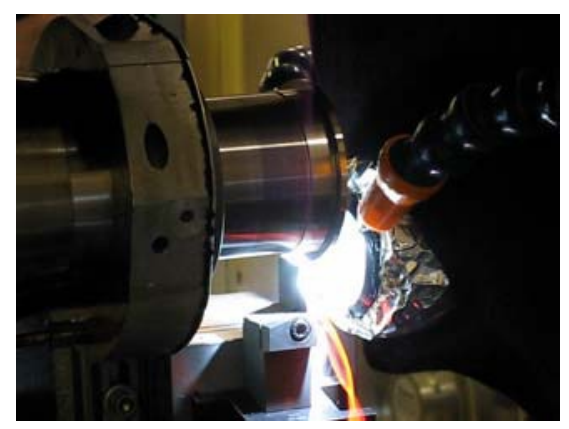

Video 1. Camcorder movie of a typical machining test. http://dx.doi.org/10.1117/12.818799.5

As with any measurement system, there are sources of uncertainty that must be understood to fully assess the quality of the measurement results. We group the sources of uncertainty into five categories: calibration of camera sensitivity and offset, conversion of apparent to true temperature, camera optics, electronic effects, and other issues. Some uncertainties are familiar to anyone trying to perform radiation thermography measurements, such as the need to calibrate the camera and to take emissivity into account. However, there are many issues unique to metal cutting due to the high magnifications, high surface speeds, and ever changing emissivity of the metal as chips form. Also, each source of uncertainty may be of greater or lesser importance depending on the specific quantity measured. For example, when 
measuring how much the work piece heats up, camera sensitivity and pixel cross talk are of major concern. By contrast, when measuring a peak temperature, size-of-source and motion blur effects are likely to be more important.

This paper focuses on thermal spectrum measurements, though visible spectrum measurements are also shown. It is not intended to be an exhaustive list of all possible uncertainty sources, but shows many qualitative examples. Images of a number of cutting experiments with different cutting conditions and materials are shown. For brevity, the specifics of the cutting experiments are not given, as this paper has a measurement focus.

\section{PROPERTIES OF MEASUREMENTS}

When reading Section 3, it is useful to remember that any temperature measurement is valid only at some location during some time interval. Thus, to fully gauge uncertainties associated with a measurement, one may need to consider three different uncertainties. Amplitude uncertainty is what most researchers typically think of as uncertainty. It refers to how well one knows the amplitude of the measurement. For example, if a measured temperature is stated as $700 \mathrm{~K} \pm 5$ $\mathrm{K}$, the amplitude uncertainty is $5 \mathrm{~K}$. Temporal uncertainty refers to how well one knows when a measurement occurred. If a measurement occurred $100 \mathrm{~ms} \pm 2 \mathrm{~ms}$ after time zero, the temporal uncertainty is $2 \mathrm{~ms}$. Spatial uncertainty refers to how well one knows the location in space of the measurement. If the position of a given pixel in an image corresponds to $100 \mu \mathrm{m}$ from the edge of a specimen $\pm 1 \mu \mathrm{m}$, the spatial uncertainty is $\pm 1 \mu \mathrm{m}$.

The goal is to determine which uncertainty sources are of most concern by first considering which are important to the question being asked. For example, if one wished to know when a peak in the average temperature occurred, uncertainty sources which affect temporal uncertainty are probably of greater concern than those which effect amplitude or spatial uncertainty. However, one must remember that one uncertainty may also affect another uncertainty. For example, suppose one wished to know the temperature $200 \mu \mathrm{m}$ from the edge of a specimen. If temperatures a short distance away have very different amplitudes (there are large thermal gradients in the image), even a modest spatial uncertainty can cause a large amplitude uncertainty, regardless of how well the sensitivity of the camera is known.

There are many other issues involved in uncertainty analysis which are described elsewhere ${ }^{[4]}$.

\section{UNCERTAINTY SOURCES}

In this paper, uncertainty sources are divided into five categories: calibration of camera sensitivity and offset, conversion of apparent to true temperature, camera optics, electronic effects, and other issues. Generalities for each category will be presented, along with specific illustrative examples.

\subsection{Calibration of camera sensitivity and offset}

The first category of uncertainty sources is related to the camera sensitivity (the slope of the line in a linear calibration curve) and offset (the intercept of the line). This category directly affects the amplitude uncertainty of the temperature measurement. Four examples are discussed below.

Example 1: Incorrect use of a blackbody. When using a blackbody to calibrate a thermal camera, it is tempting to assume the emissivity of the blackbody is 1 . However, consider the case of imaging two blackbodies, each set to $800{ }^{\circ} \mathrm{C}$, with a camera sensitive to $4 \mu \mathrm{m}$ wavelength light. The first blackbody has an emissivity of 0.95 (typical for a plate blackbody), with a radiance temperature of $784^{\circ} \mathrm{C}$, while the second blackbody has an emissivity of 0.99 (typical for a spherical cavity blackbody), with a radiance temperature of $797{ }^{\circ} \mathrm{C}$. The apparent $13{ }^{\circ} \mathrm{C}$ disagreement is due solely to the difference in emissivities. Not taking emissivity into account can lead to significant errors, even when emissivity is close to 1 .

Example 2: Sensitivity to polarization. Another source of uncertainty is the dependency of camera sensitivity to polarization of the measured thermal radiation. For polarization to be a significant source of uncertainty, the thermal radiation measured must be at least partially polarized and the sensitivity of the camera system must vary as a function of polarization angle. 
To estimate uncertainty, we will only consider linear polarization and ignore circular polarization. That is, we will assume that $S_{3}$ of the Stokes vector ${ }^{[5]}$ is 0 . The sensitivity of our camera system to polarization was estimated by imaging a black body through a linear polarizer rotated at various angles. For our system, there is approximately a $\pm 5 \%$ variation in intensity. Table 1 shows errors which result from a $\pm 5 \%$ variation in intensity at various apparent temperatures.

\begin{tabular}{|c|c|c|}
\hline $\begin{array}{c}\text { Apparent } \\
\text { temperature } \\
\left({ }^{\circ} \mathrm{C}\right)\end{array}$ & $\begin{array}{c}\text { Error when all light is linearly } \\
\text { polarized in the sensitive direction } \\
\left({ }^{\circ} \mathrm{C}\right)\end{array}$ & $\begin{array}{c}\text { Error when all light is linearly polarized } \\
\text { perpendicular to the sensitive direction } \\
\left({ }^{\circ} \mathrm{C}\right)\end{array}$ \\
\hline 400 & +6.5 & -7.0 \\
\hline 500 & +8.6 & -9.0 \\
\hline 600 & +10.9 & -11.1 \\
\hline 700 & +13.3 & -13.7 \\
\hline 800 & +16.1 & -16.4 \\
\hline 900 & +18.9 & -19.2 \\
\hline 1000 & +21.9 & -22.3 \\
\hline
\end{tabular}

Table 1. Errors at various apparent temperatures resulting from a $\pm 5 \%$ variation in intensity.

There are a variety of reasons to suspect that the light observed during machining might be partially polarized. One is the fact that when a surface is viewed off-axis, both reflected and emitted light may be polarized ${ }^{[6-8]}$. In fact, a polarizer may be used to reduce the effect of reflections in thermal imaging ${ }^{[7]}$. Ongoing research involves imaging the machining process through a rotating linear polarizer to see if there is a significant amount of polarization.

Example 3: An incomplete understanding of resolution and repeatability. Briefly stated, resolution is the smallest possible difference between two values of the measurand that can be measured and repeatability is the range of values attained by repeated measurements under the same conditions. The intensity value at any pixel in an image is a digitized representation stored as binary Digital Levels, or DLs. Since intensity is acquired in DLs, one may argue that the best possible resolution is the intensity corresponding to the least significant bit of a DL value.

However, even if the response of a camera is a linear function of intensity, it is not a linear function of temperature, and thus resolution of the temperature measurement depends on the temperature being measured. Figure 3 illustrates this. If one wished to write specifications for a camera, one could specify that for a range of $10{ }^{\circ} \mathrm{C}$ to $290{ }^{\circ} \mathrm{C}$, the resolution is $0.6{ }^{\circ} \mathrm{C}$ or better, or that for a range of $100{ }^{\circ} \mathrm{C}$ to $290{ }^{\circ} \mathrm{C}$, the resolution is $0.1{ }^{\circ} \mathrm{C}$ or better. While the two specifications seem very different, they actually describe the same camera. Thus, any comparison of cameras or camera settings must be over the same temperature range. Also note that within the same thermal image, hotter portions have a finer temperature resolution than colder portions. A similar argument may be applied to repeatability.

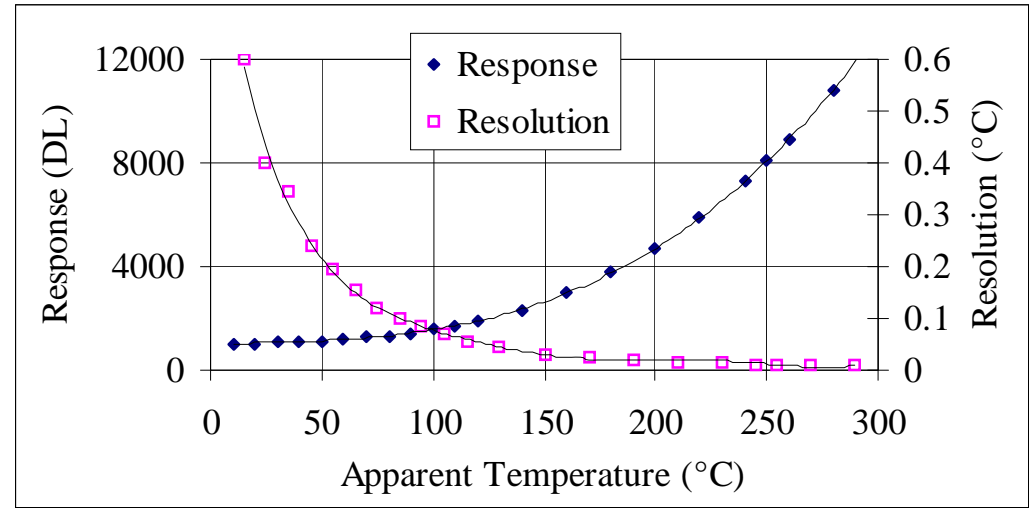

Fig. 3. Response of a camera in DLs, and resulting resolution, as a function of temperature. 
Example 4: Not considering the range of linear operation. Figure 4a shows a situation with two desirable characteristics. First, only the linear range of the camera is used. Second, the entire linear range is used, not a small section of it. Figure $4 \mathrm{~b}$ shows just the lower portion of the same curve.

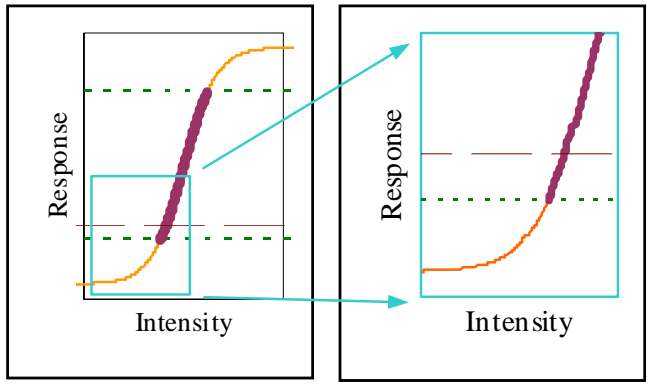

a

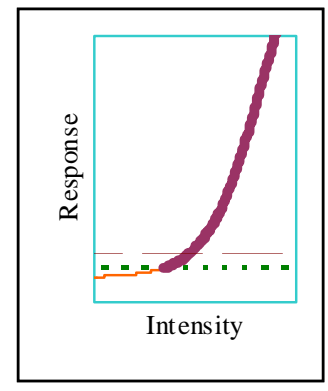

c

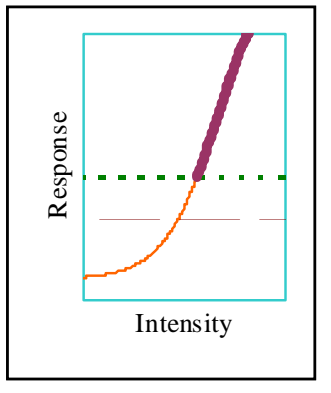

d

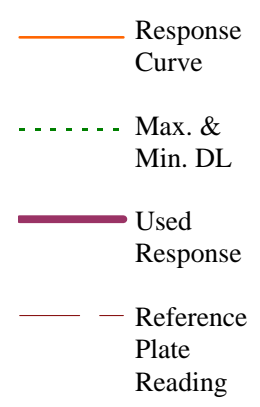

Reading

Fig. 4. Idealized camera response. $4 \mathrm{a}$ and $4 \mathrm{~b}$ show an ideal situation while $4 \mathrm{c}$ and $4 \mathrm{~d}$ are problematic.

A formula describing the response of the camera system is

$$
C=E_{e}+R\left(E_{o}+I\right),
$$

where $C$ is the total camera response, $E_{e}$ is the error due to electrical effects such as voltage offsets, function $R$ is the response of the camera to light, $E_{o}$ is the error due to optical effects such as infrared light emitted by the optical system, and $I$ is the intensity of light emitted by the target being measured. To remove both optically and electronically induced offsets in $C$, it is common practice to use a room temperature "reference plate" to perform a single point "calibration" of the camera. If the camera is used only in the linear portion of the response, then

$$
R\left(E_{o}+I\right)=R\left(E_{o}\right)+R(I)
$$

and Equation 1 may be restated as

$$
C=E_{e}+R\left(E_{o}\right)+R(I)=E_{c}+R(I),
$$

where $E_{c}$ is the combined error due to electronic and optical effects. Subtracting a constant, such as a reference plate reading, works in this case.

As shown in Figure 4c, a researcher may wish to extend the dynamic range of the camera by deliberately using the camera beyond the linear range. However, as a result, Equation 2 and Equation 3 might not apply and errors could result. This does not mean that the dynamic range of the measurement can not be extended by using both the linear and nonlinear portions of the response. It does mean that methodologies used when making the measurement need reevaluation.

Figure $4 \mathrm{~d}$ shows another potentially problematic situation. Here, the camera is operated within the linear range, but the reference plate is at too low a temperature (intensity) and the measured digital reading is 0 (zero) DLs. Imagine a scenario where the temperature of the optics increases or decreases, causing them to emit more or less light. This causes the intensity of light received by the camera sensor while measuring the reference plate to change. However, the camera only reports zeroes and thus the change is not compensated for. This error may be avoided by using a reference plate at a high enough temperature that the camera yields DLs above zero.

\subsection{Conversion of apparent to true temperature}

Once a thermal image is acquired and converted to apparent temperatures, the next step is to convert apparent to true temperatures. In this paper, radiance temperature is the temperature predicted by Planck's equation ${ }^{[6]}$ without taking reflections into account. By contrast, apparent temperature includes both light emitted by and reflected from the body being measured. The conversion of apparent to true temperature is the second category of uncertainty sources considered. This category directly affects the amplitude uncertainty of the temperature measurement. While uncertainties in emissivity generally dominate, other issues may be important as well. The three examples are discussed next. 
Example 1: Non-uniform surface textures. To measure the emissivity of the side of the chips imaged during machining, two chips are placed in a pin vise with a thermocouple sandwiched between them. Shown in Figure 5, thermal tweezers are then used to heat the chips to a known temperature. The chips are imaged by the dual spectrum system and the difference between the thermocouple and apparent temperature is attributed to local emissivity $\varepsilon$ of the chip surface. Due to the extreme deformation involved, chips typically have a much rougher surface than the workpiece. Thus, one expects the chips to be more emissive than the workpiece.

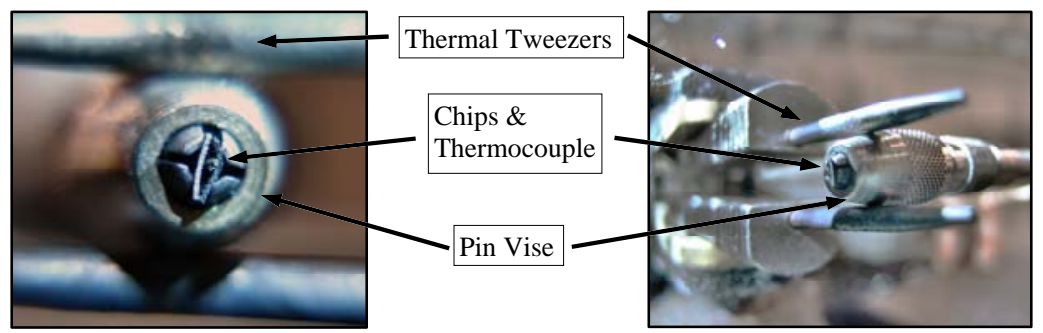

Fig. 5. Two chips are heated to a known temperature to estimate their emissivity.

Figure 6 shows a visible (6a) and thermal (6b) spectrum image of a segmented chip heated as shown in Figure 5. The relatively planar portion of each segment we call the face of the segment. The area between segments we call the gap. A chip often has some areas of side flow where metal has flowed toward the camera. The side flow is often out of focus due to the limited depth of field of the camera system. Emissivity $\varepsilon \approx 0.3$ for the segment faces while $\varepsilon \approx 0.5$ for the gap and side flow portions of the image. It is not uncommon for emissivity values to vary from 0.2 to 0.6 on a single segment face. With such large variation in emissivity, a large temperature uncertainty is difficult to avoid.

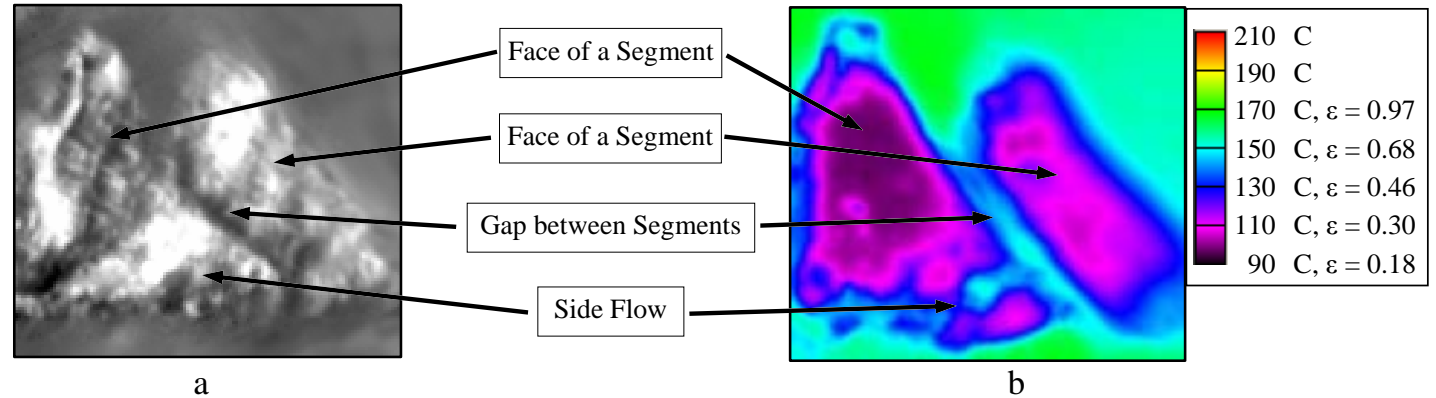

Fig. 6. A visible (6a) and thermal spectrum image of apparent temperatures with corresponding emissivity values (6b).

The $172{ }^{\circ} \mathrm{C}$ chip is about $0.6 \mathrm{~mm}$ thick (up and down in the image).

Video 2 shows a gap between segments during machining. It appears to be much hotter than the chip itself. Is that true, or has the gap formed a micro-blackbody, raising the effective emissivity? The lowest apparent temperature detectable at these camera settings is $230{ }^{\circ} \mathrm{C}$. Using an emissivity value of 0.3 (from Figure 6b), the hottest true temperature possible for the segment faces is about $345^{\circ} \mathrm{C}$. Using an emissivity value of 0.5 for the gap (also from Figure $6 \mathrm{~b}$ ), the true temperature of the gap is somewhere between $370{ }^{\circ} \mathrm{C}$ and $510{ }^{\circ} \mathrm{C}$. If one had assumed an emissivity of 0.3 for the gap, a true temperature between $440{ }^{\circ} \mathrm{C}$ and $620^{\circ} \mathrm{C}$ would have been computed instead. Thus, the gap is actually hotter than the face, but not by as much as one would have predicted if the non-uniform emissivity was not correctly accounted for.

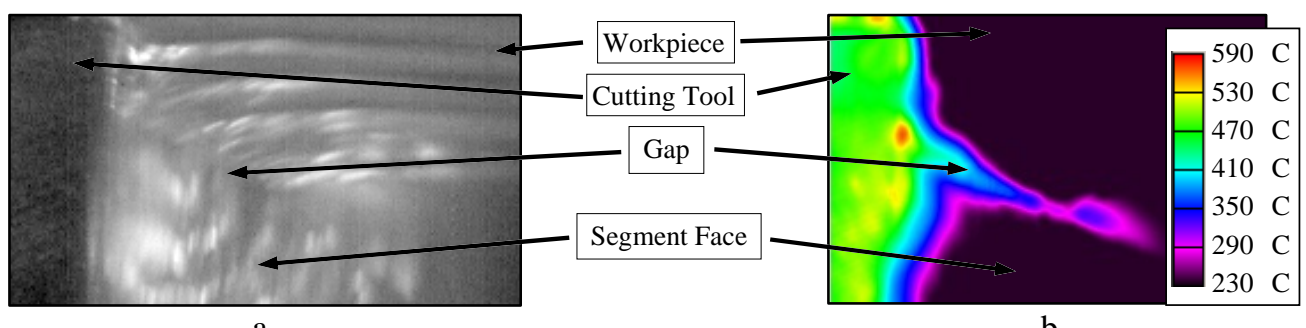

$\mathrm{a}$

$\mathrm{b}$

Video 2. A synchronized high-speed visible (a) and medium-speed thermal (b) spectrum movie of segmented chip formation. The thermal movie shows apparent temperature. http://dx.doi.org/10.1117/12.818799.6 
Some researchers attempt to avoid issue of non-uniform emissivity by using a long integration time while imaging chip formation. The motion of the chip causes each pixel of the resulting images to be an average of many locations on the chip surface. It is then claimed that this yields an average temperature. However, while each pixel may represent an average intensity, it does not yield an average (arithmetic mean) temperature. This is because the equation converting intensity to temperature is highly non-linear.

Example 2: The form of the equation used to convert to true temperature. There are multiple modifications of Planck's equation in the literature. Some take into account effects such as reflectivity, transmittance, atmospheric absorption, multiple reflections, changes in emissivity as a function of temperature, or whether to use a single wavelength or integrate over a range of wavelengths. Also, some are more numerically well-behaved (less susceptible to round-off errors) than others. The form we use is

$$
S=\left|\left(\int_{\lambda_{1}}^{\lambda_{2}} \frac{w_{\lambda} / \lambda^{5}}{e^{\frac{c_{2}}{\lambda T_{\text {apparent }}}}-1} d \lambda\right)-\left(\varepsilon \int_{\lambda_{1}}^{\lambda_{2}} \frac{w_{\lambda} / \lambda^{5}}{e^{\frac{c_{2}}{\lambda T_{\text {true }}}}-1} d \lambda\right)-\left((1-\varepsilon) \int_{\lambda_{1}}^{\lambda_{2}} \frac{w_{\lambda} / \lambda^{5}}{e^{\frac{c_{2}}{\lambda T_{\text {environment }}}}-1} d \lambda\right)\right|,
$$

where $\lambda$ is the wavelength of light, $\lambda_{1}$ and $\lambda_{2}$ are the shortest and longest wavelength the camera is sensitive to respectively, $w_{\lambda}$ is the relative sensitivity of the camera at wavelength $\lambda, \varepsilon$ is the emissivity of the target, $c_{2}$ is the second radiation constant $(14387 \mu \mathrm{m} \cdot \mathrm{K}), T_{\text {apparent }}$ is the apparent temperature of the target as measured by the camera, $T_{\text {environment }}$ is the temperature of the surrounding environment, and $T_{\text {true }}$ is the true temperature of the target. An unknown quantity may be determined by substituting known values and adjusting the unknown value until $S$ is minimized. This works well for determining $T_{\text {apparent }}, T_{\text {true }}$, or $\varepsilon$. Solving for $T_{\text {environment }}$ often results in a large uncertainty.

Most researchers use a filter to ensure that a single, well known wavelength is detected by the camera. However, this significantly reduces the sensitivity of the camera, necessitating the use of longer integration times, causing significant motion blur and washing out most of the detail in the thermal images. This contrasts with the thermal images in Video 2, which were acquired with no filters and an integration time of $10 \mu \mathrm{s}$. The motion blur is only a few pixels, preserving most of the detail. However, to accurately convert apparent to true temperature, the camera sensitivity as a function of wavelength must be known. Table 2 shows an example of how computed true temperature is affected by the assumptions made for our camera. Whether a single wavelength or a range of wavelengths are used makes a significant difference.

\begin{tabular}{|l|c|c|c|}
\hline \multicolumn{1}{|c|}{ Assumption about $\lambda$} & $\lambda_{l}(\mu \mathrm{m})$ & $\lambda_{2}(\mu \mathrm{m})$ & $\mathrm{Computed} T_{\text {true }}\left({ }^{\circ} \mathrm{C}\right)$ \\
\hline Single camera nominal (average of camera nominal high and low) & 4.35 & 4.35 & 1086 \\
\hline High and low camera nominal & 3.6 & 5.1 & 1077 \\
\hline Single measured (average of high and low measured response of entire system) & 4.45 & 4.45 & 1092 \\
\hline Measured response of entire system & 3.8 & 5.1 & 1086 \\
\hline
\end{tabular}

Table 2 Various values of $T_{\text {true }}$ resulting from different assumptions about $\lambda$, where $T_{\text {apparent }}=800{ }^{\circ} \mathrm{C}$, $T_{\text {environment }}=20^{\circ} \mathrm{C}, \varepsilon=0.5$, and $w_{\lambda}=1$ for all $\lambda$.

Example 3: Oxide layer changes. In Figure 5 and Figure 6, chip emissivity was determined by measuring chips after the test. Figure 7 shows two chips from the same test. Note that, despite being from the same test, there is a significant color difference due to their oxide layers. The top most chip has an average emissivity of 0.4 , compared to 0.8 for the chip on the bottom. Which value should be used? If the oxide layer did not form until after the chip had left the field of view of the thermal camera, perhaps neither is correct!

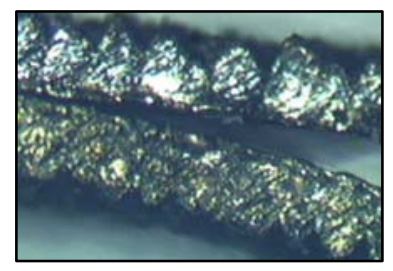

Fig. 7. Two chips about $0.6 \mathrm{~mm}$ thick (vertical in this image) from the same cutting test, but with different oxide layers. 
Video 3 illustrates the fact that oxidation is a reaction that takes time to occur and is sometimes exothermic (produces heat). These two video streams, acquired from two different vantage points at the same moments in time, show a very thin chip that is initially not hot enough to visibly glow. Milliseconds later, the chip is hot enough to glow very brightly. As shown in Video 4, the once solid chip sometimes melts and boils well after the test is finished. The reason the temperature rise is not obvious with the normal, much thicker chips is that thick chips have a lot more metal to absorb the exothermic heat energy. It should be noted that oxidation is not always exothermic. It may also be either endothermic (receives heat from the surroundings) or temperature neutral.

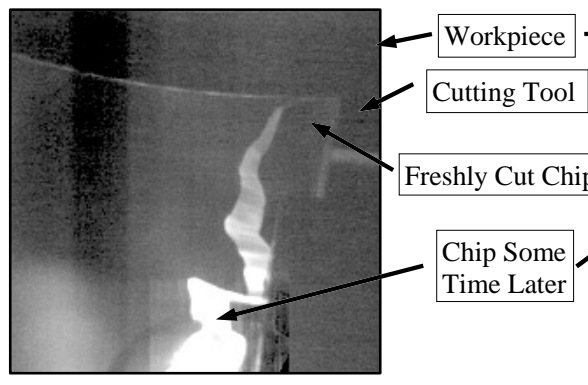

a

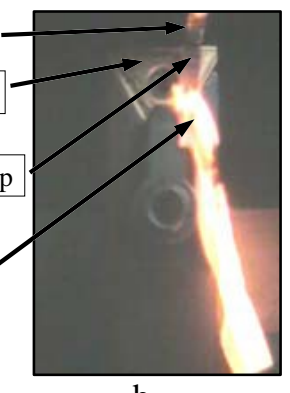

b

Video 3. A low-magnification high-speed visible light video (3a) and an even lower magnification camcorder video (3b) of a very thin chip, less than $0.1 \mathrm{~mm}$ thick, illustrating exothermic oxide formation. http://dx.doi.org/10.1117/12.818799.7

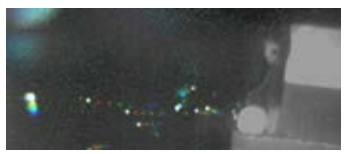

Video 4. A low-magnification high-speed visible light video of a very thin chip, less than $0.1 \mathrm{~mm}$ thick. The chip has completely melted and boils violently, even after the test is over. The video is colorized to highlight motion. http://dx.doi.org/10.1117/12.818799.8

One consequence of these oxide layer effects is that researchers must use care when using chips measured after the test to estimate emissivity of the chip when imaged during the test.

\subsection{Camera optics}

No optical system yields perfect images under all conditions. An image may be distorted, or portions may be out of focus (as when there is extreme side flow). The optical system directly affects both amplitude and spatial uncertainty of the temperature measurements. An example is optical pixel crosstalk, discussed next.

Optical pixel crosstalk. Especially when there is a high ratio between the brightest and darkest areas of interest, unwanted scattering and reflections in the imaging system cause intensity values of some pixels to apparently affect intensity values of other pixels. Figure 8a shows a simple experiment designed to gauge this crosstalk. The thermal camera images a chilled, $500 \mu \mathrm{m}$ wide aperture. On the far side of the aperture, a removable foil blocks a hot blackbody. The camera acquisition is started with as high a frame rate as is practical. The foil is then rapidly removed. The thermal movie is examined and a frame just before (Figure 8b) and just after (Figure 8c) foil removal is compared.

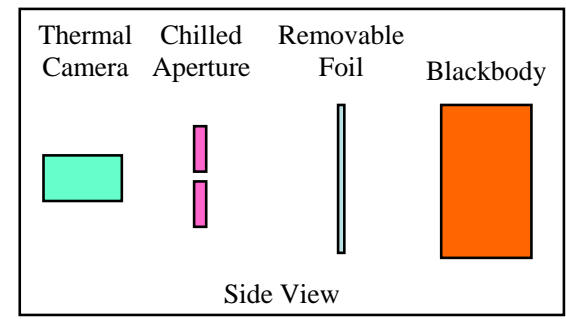

a

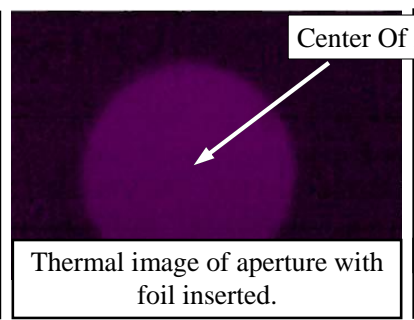

$\mathrm{b}$

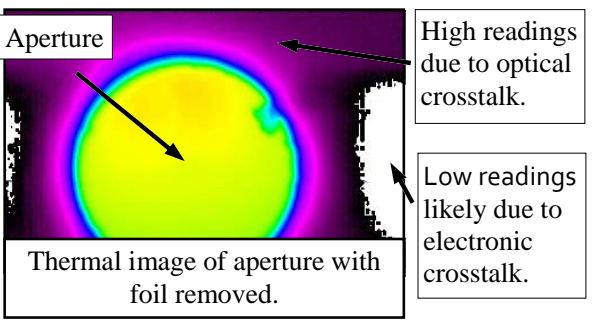

c

Fig. 8. A simple experiment used to gauge optical and electronic pixel crosstalk. Two images are compared, one with and one without the foil (which acts like a shutter). Electronic crosstalk is discussed in the next section. 
The reason for the rapid foil removal and high frame rate is to minimize the amount of time between foil removal and acquisition of the second image. This reduces the possibility that the actual aperture temperature is affected by radiative heating from the blackbody.

As expected, the temperature in the center of the aperture is very high. However, the area around the aperture has also increased due to scatter in the optical system. Notice that in the center of the left and right sides of the image, the apparent temperature has actually decreased. While this theoretically could be caused by the Optical Transfer Function of the system going negative ${ }^{[8]}$, it is more likely due to electronic pixel crosstalk, which is discussed in the next section.

\subsection{Electronic effects}

A variety of uncertainty sources are associated with the electronics within the camera. They directly affect amplitude and temporal uncertainty in temperature measurements. Four examples are described below.

Example 1: Electronic pixel cross talk. A sensor array where each pixel has its own power supply lines and analog to digital converter is simply not practical. Thus, pixel elements within a camera share electronic components. Figure 9 shows a simplified, stylized eight-pixel camera sensor. Any power supply line has some resistance. The power supply lines on sensor array chips are very thin, and thus the resistance is high when compared to a normal copper wire. If the current is low, the voltage drop across the supply line is negligible. However, if a pixel element is exposed to a very bright intensity, it may draw more current than normal. This could cause a drop in the voltage supplied to other pixel, affecting their reading. In our eight-pixel sensor, an overdriven pixel $\mathrm{P}_{1}$ could affect pixel $\mathrm{P}_{2}$. If $\mathrm{P}_{1}$ is severely overdriven, or saturated, it often takes time before it behaves normally again. This is sometimes referred to as the saturation recovery time. A similar situation arises from the fact that groups of pixels share a multiplexer and an analog to digital converter. The end result is that a few very bright (hot) pixels in an image may induce large errors in the dimmer (cooler) ones.

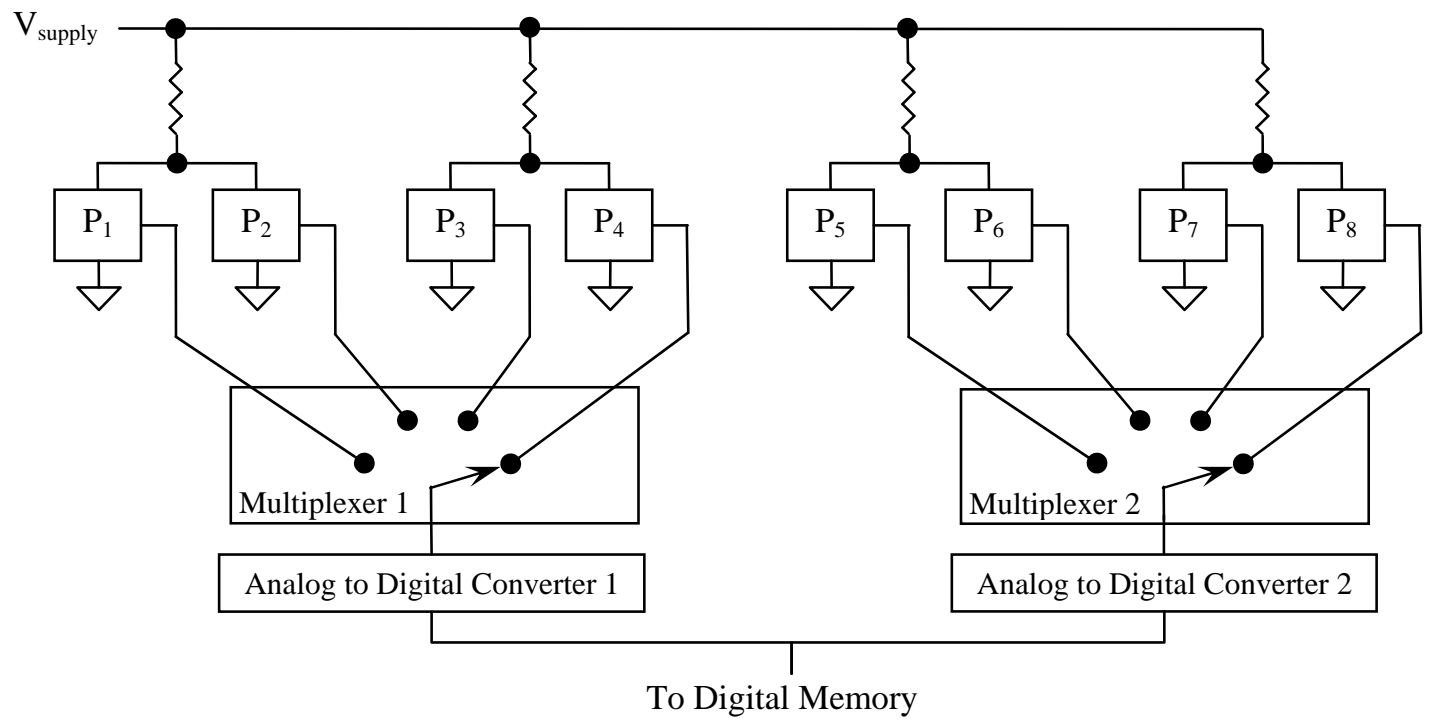

Fig. 9. Electronic pixel cross talk for a simplified, stylized eight-pixel $\left(\mathrm{P}_{1}-\mathrm{P}_{8}\right)$ camera sensor.

Example 2: Nonuniformity. Each pixel on a sensor array has a different sensitivity to light. Also, the amplifiers in the analog to digital converters in Figure 9 may have slightly different gains and offsets. This is especially evident at short integration times, where nonuniformity becomes more severe. The process of correcting for these effects is called a NonUniformity Correction, or NUC. There are many schemes for performing a NUC ${ }^{[9-16]}$. Some are quite complex, and it is not always obvious how they effect camera calibration. We calibrate each pixel individually, so a separate NUC is not required.

Example 3: Timing errors. There are several types of timing errors. One type, sometimes referred to as timing jitter, is when there is large variation in either the time intervals between frames or the integration times of the frames. Another type is the phenomenon of dropped frames. This is where the hardware is unable to process the video stream rapidly, resulting in images from the stream becoming lost. 
Figure 10a shows a data acquisition scheme typical in high-speed visible light cameras. Because the digital memory is dedicated to data acquisition, it does a good job of storing frames at a high rate of speed. Also, having the memory connected directly to the sensor improves speed as the long cable connecting the camera to the computer is often a bandwidth bottleneck. The stream is saved to the computer hard drive after the test is over.

This scheme contrasts with the scheme shown in Figure 10b, which is common in thermal cameras, as well as mediumspeed and slow-speed visible light cameras. Here, a general purpose computer is heavily relied upon to capture the stream in real time. If the camera outputs frames faster than either the computer memory or hard drive can process them, frames will be lost. Each lost (dropped) frame corresponds to a timing jitter of $100 \%$ (100\% error). Even with a fast computer, the scheme in Figure 10a typically outperforms the scheme in Figure 10b.

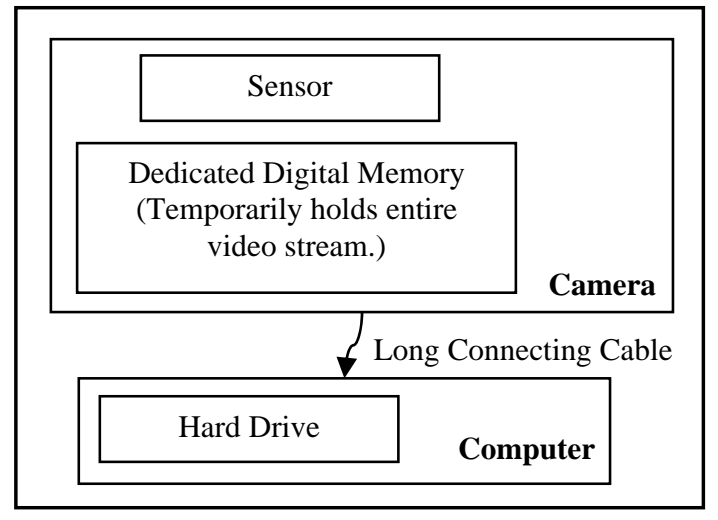

a

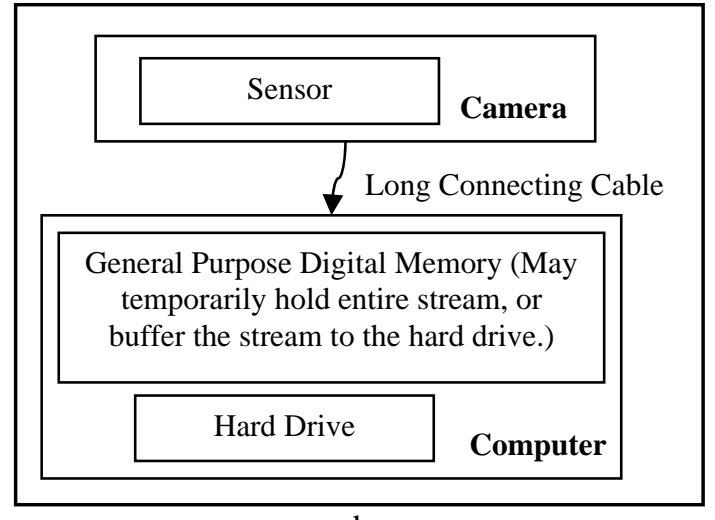

b

Fig. 10. Two hardware schemes for acquiring video streams.

Example 4: Persistence. As previously discussed, if a pixel is exposed to very bright light and saturates, it takes time to recover. In addition, even when not saturated, all electronic devices have a limit on how fast they can change their output voltage or current. This is especially easy to detect in a high-speed visible light camera set to either a high gain or a low gamma (high gain at low light levels). It is likely that thermal cameras are affected in a similar way. Figure 11 shows four visible light images of a backlit, $200 \mu \mathrm{m}$ diameter aperture mounted near the edge of a rotating wheel. Two surface speeds and two integration times are shown. Figure 11d shows both motion blur (discussed in the next section) and persistence.

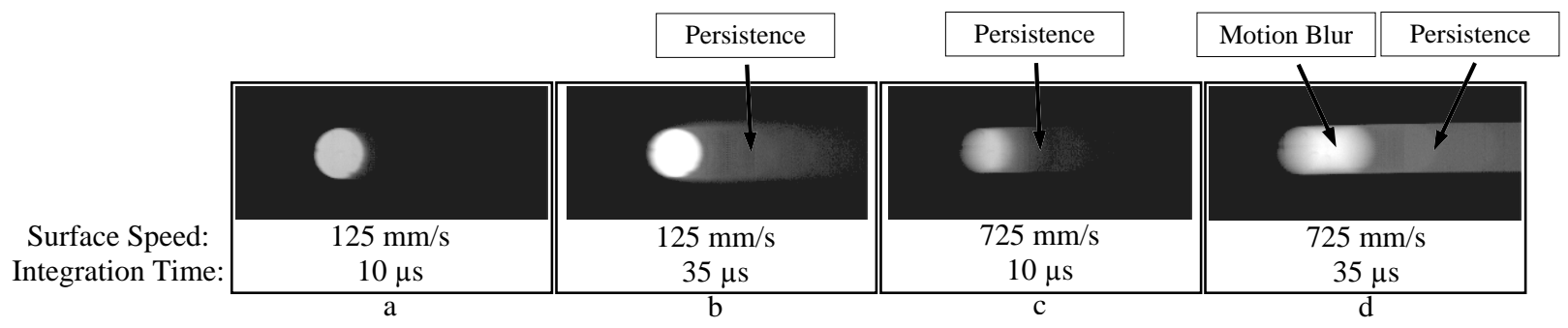

Fig. 11. Visible light images of a backlit $200 \mu \mathrm{m}$ diameter aperture mounted near the edge of a rotating wheel.

\subsection{Other issues}

There are uncertainty sources not discussed above. They may directly affect amplitude, temporal, and spatial uncertainty in temperature measurements. Two examples are given below.

Example 1: Motion blur. An example of motion blur may be seen by comparing Figure 11b to Figure 11d. In Figure 11b, there is little motion blur and we see the expected disk shape. In Figure 11d, the disk shape is spread out into a larger shape. This not only distorts the disk, but makes it appear dimmer than it actually is. Had this been a thermal image, significant error in the measured temperature would have resulted. 
Example 2: The inherent difficulty of measuring very small features. When imaging the metal cutting process, many features, such as the thickness of the shear zone, may be in the range of only $0.1 \mu \mathrm{m}$ to $10 \mu \mathrm{m}$ thick. A variety of factors makes measuring the temperatures of such small features problematic. One factor is that spatial resolution is limited by the wavelength of light used to image the target ${ }^{[8]}$. This is one reason why visible light cameras can image smaller features than thermal cameras. Also, if a feature is so small that it does not completely fill a pixel, then the amplitude will be incorrect ${ }^{[8]}$. Finally, there may be diffraction effects ${ }^{[8,17]}$.

\section{CONCLUSIONS}

Dual-spectrum imaging is a powerful technique for enabling a better understanding of the metal cutting process. However, many uncertainty sources need to be understood to correctly acquire and interpret the data. In this paper, uncertainty sources were divided into five categories: calibration of camera sensitivity and offset, conversion of apparent to true temperature, camera optics, electronic effects, and other issues. Generalities for each category were presented, along with specific illustrative examples. To determine whether an uncertainty source may affect any given measurement, one must keep in mind that temperature measurements describe a temperature at a location over some time interval. Thus, any measurement has several related uncertainties: amplitude, temporal and spatial. Each uncertainty may interact to affect the final uncertainty of the measurement.

\section{ACKNOWLEDGEMENTS}

The author wishes to acknowledge Robert Ivester, Jarred Heigel, April Cooke, Johannes Soons, and Howard Yoon of the National Institute of Standards and Technology.

\section{REFERENCES}

[1] Whitenton, E., Ivester, R., Yoon, H., "Simultaneous Visible And Thermal Imaging Of Metals During Machining" Proc. SPIE Vol. 5782, pp. 71-82, Thermosense XXVII, March 2005; G. Raymond Peacock, Douglas D. Burleigh, Jonathan J. Miles; Eds.

[2] Heigel, J., Ivester, R., Whitenton, E., "Cutting Temperature Measurements of Segmented Chips using DualSpectrum High-Speed Microvideography" Transactions of NAMRI/SME, volume 36, pp. 73-80, May 20, 2008.

[3] Whitenton, E., Heigel, J., Ivester, R., "Measurement and Characterization of Dynamics in Machining Chip Segmentation" 11th CIRP International Workshop on Modeling of Machining Operations, Gaithersburg, MD, pp. 237-246, Sept 16-18, 2008.

[4] Taylor, B., Kuyatt, C., "Guidelines for Evaluating and Expressing the Uncertainty of NIST Measurement Results" NIST Technical Note 129, 1994 Edition.

[5] Distl, R., Egeler, T., "Polarization Measurement of Light Radiation" Instrument Systems, Germany, http://www.i-s.de/applications/downloads/polarization_applicationnote.pdf.

[6] DeWitt, D.P., Nutter, G. D., [Theory And Practice Of Radiation Thermometry], John Wiley \& Sons, Inc, 1988.

[7] Vollmer, M., Henke, S., Karstädt, D., Möllmann, K. P., Pinno, F., "Identification and Suppression of Thermal Reflections in Infrared Thermal Imaging” InfraMation 2004 Proceedings, ITC 104 A 2004-07-27.

[8] Holst, G. C., [Holst's Practical Guide to Electro-Optical Systems] JCD Publishing, 2003.

[9] Hayat, M. M., Torres, S. N., Armstrong, E., Cain, S. C., Yasuda, B., "Statistical Algorithm for Nonuniformity Correction in Focal-Plane Arrays" Applied Optics, Vol. 38, No. 8, pp. 772-780, March 10, 1999.

[10] Harris, J. G., Chiang, Y., "Nonuniformity Correction of Infrared Image Sequences Using the Constant-Statistics Constraint" IEEE Transactions On Image Processing, Vol. 8, No. 8, pp. 1148-1151, August 1999.

[11] Cohen, M., Cauwenberghs, G., "Floating-Gate Adaptation for Focal-Plane Online Nonuniformity Correction" IEEE Transactions On Circuits And Systems - II: Analog And Digital Signal Processing, Vol. 48, No. 1, pp. 83-89, January 2001.

[12] Ratliff, B. M., Hayat, M. M., "An Algebraic Algorithm for Nonuniformity Correction in Focal-Plane Arrays" Journal of the Optical Society of America, Vol. 19, No. 9, pp. 1737-1747, September 2002.

[13] Torres, S. N., Pezoa, J. E., Hayat, M. M., "Scene-Based Nonuniformity Correction for Focal Plane Arrays by the Method of the Inverse Covariance Form" Applied Optics, Vol. 42, No. 29, pp. 5872-5881, October 10, 2003.

[14] Ratliff, B. M., Hayat, M. M., Tyo, J. S., "Radiometrically Accurate Scene-Based Nonuniformity Correction for Array Sensors" Journal of the Optical Society of America, Vol. 20, No. 10, pp. 1890-1899, October 2003. 
[15] Zhou, H., Liu, S., Lai, R., Wang, D., Cheng, Y., "Solution for the Nonuniformity Correction of Infrared Focal Plane Arrays" Applied Optics, Vol. 44, No. 15, pp. 2928-2932, May 20, 2005.

[16] Pezoa, J. E., Hayat, M. M., "Multimodel Kalman Filtering for Adaptive Nonuniformity Correction in Infrared Sensors" Journal of the Optical Society of America, Vol. 23, No. 6, pp. 1282-1291, June 2006.

[17] Shirley, E. L., "Revised Formulas for Diffraction Effects with Point and Extended Sources" Applied Optics, Vol. 37, No. 28, pp. 6581-6590, October 1998. 